are fever, dysuria, frequency, urgency, suprapubic tenderness and haematuria. Symptoms indicating upper UTI are loin pain, flank tenderness, fever and rigors. Common causative organisms are Escherichea coli followed by Klebsiella, Enterococci, Proteus, Coagulase negative staphylococci and Staphylococcus saprophyticus. If not managed adequately UTI has been considered a risk factor for the development of renal insufficiency, scarring and end stage renal disease in children. UTI may be suspected on the basis of clinical features or findings on urinalysis or both. A urine culture is necessary for confirmation and appropriate therapy. Nitrites and leukocyte esterase are usually positive in infected urine, WBC count $>100 /$ $\mathrm{cmm}$ is highly suggestive of UTI.

Aim This audit aims to assess sensitivity and resistance of Ecoli causing UTI in children admitted to Mayo University Hospital where children with presumed UTI are empirically treated with co-amoxiclav as first line therapy until culture and sensitivity results are available. The level of resistance to Co-Amoxiclav has been increasing over the years.

Methodology Retrospective cross sectional hospital based study of children admitted to the Paediatric Ward in Mayo University Hospital, with a primary diagnosis of UTI over a period of 12 months from the beginning of July 2016 to the end of June 2017.The list of patients with the diagnosis of UTI was obtained from the HIPE department of the hospital. Charts of children from birth up to the age of 15 years were reviewed.

Results During the study period, there were 93 admissions to the hospital with UTI. Children from 1-5 years of age were the most affected age group (39.8\%) followed by those less than 1 year old $(36.6 \%)$, females accounted for $(74 \%)$ of cases. Fever $(78.5 \%)$ and irritability $(47 \%)$ were the most common presenting symptoms. The most common pathogen was E.coli (76\%) followed by Pseudomonas and Proteus. Almost (44\%) of cases of E.coli UTI were resistant to CoAmoxiclav.

Conclusion There is high level of E.coli resistance to CoAmoxiclav in our region. According to microbiology recommendations, a resistance of $20 \%$ is significant enough to warrant a change of practice. Due to high resistance in our cohort that has emerged over the last few years we advise updating departmental protocol and adding a second antibiotic.

\section{GP120 BRONCHO-OBSTRUCTIVE SYNDROME IN EARLY CHILDHOOD. THE BREASTFEEDING AS A PROTECTIVE AND THE ANTIBIOTIC USE THROUGH THE FIRST YEAR OF LIFE AS A PREDISPOSING FACTOR}

Elena Georgieva*. University Childrens Hospital, Sofia, Bulgaria

\subsection{6/archdischild-2019-epa.185}

The broncho-obstructive syndrome/BOS/is a common health problem in the early childhood. The frequency of 'wheezing' in infancy and its etiopathogenetic factors are very important for the likelihood of development of asthma.

The present research includes 131 children with 'wheezing' before the age of three years and 74 healthy children without similar symptoms.

The results of complex study of 33 etiopathogenetic factors for the development of BOS syndrome in early childhood shows 10 of them as most significant, including cow's milk allergy, breastfeeding duration, period of introduction of milk formula, application of antibiotic through the first year of life.

The study confirms the importance of breastfeeding as a protective factor against the development of BOS in infancy/ $\mathrm{p}<0,05 /$.

It shows also the role of the early introducing of nutrition with milk formula/p $<0,1 /$ and the antibiotic treatment throughout the first year of life/p $<0,001 /$ as a predisposing factors for the occurrence of BOS.

The role of cow's milk allergy for the development of $\mathrm{BOS} / \mathrm{p}<0.1 /$ shows the importance of breastfeeding and avoidance of the early introducing of milk formula too.

Exsept all other positive influences the group of the breastfeeding children needs signifiacant less antibiotic use compared to the others $/ \mathrm{p}<0,005 /$.

In conclusion the long term of breastfeeding, the avoidance of the early introducing of milk formula and antibiotic use may help to reduce the early childhood 'wheezing' and probably its conversion into asthma later.

\section{GP121 MAKE A LIST, CHECK IT TWICE: IMPROVING WRITTEN COMMUNICATION IN PAEDIATRIC OUTPATIENT DEPARTMENTS}

${ }^{1}$ Miriam Smyth*, John Fitzsimons ${ }^{2}$. 'Our Lady of Lourdes, Hospital Drogheda, Drogheda, Ireland; '2Our Lady of Lourdes Hospital, Drogheda, Co. Louth, Drogheda, Co. Louth, Ireland

\subsection{6/archdischild-2019-epa.186}

Aims This quality improvement initiative aimed to improve the quality of paediatric out-patient department (OPD) letters in a non-tertiary centre, by assessing adherence to a predesigned layout. The Sheffield Assessment Instrument for Letters (SAIL) is an assessment tool that gauges written communication performance in the OPD setting. This pre-designed lay out has been proven to improve the quality of clinic letters. We assessed its efficacy and looked at the uptake of this template over a year long period.

Method Clinic letters generated from consultant led general paediatric clinics over one year were retrospectively analysed via a hospital database. A checklist based on SAIL assessment tool was designed. This modified checklist included the following parameters; word count, NCHD grade, use of paragraphs, problem list. All doctors starting or continuing to work in the paediatrics outpatients department were introduced to the template system at the beginning of their clinic. We compared our numbers between each of the four interventions which included; education sessions and laminated template placement in each clinic room to assess our progress. We performed a comparative analysis on the uptake of this intervention following its initial introduction in January 2018 and its continued use from July 2018. Our main focus was on inclusion of problem lists and medication lists in the GP letter

Results Initial assessment: 51\% $(\mathrm{n}=132)$ of the letters included problem lists. $19.3 \% \quad(n=50)$ included medication lists. Post first intervention: $77 \%(\mathrm{n}=94)$ had problem lists and $47 \%$ $(\mathrm{n}=57)$ had medication lists. A template was placed in all clinic rooms.

A year on: $78.9 \%(n=94)$ had problem lists. 31\% $(n=38)$ had medication lists. A repeat education session took place. Post third intervention: $88 \%(\mathrm{n}=208)$ had problem lists and $58 \%(n=136)$ had medication lists. Over all there was a 
$37 \%$ increase in the inclusion of problem lists and $12 \%$ increase in medication list inclusion.

Conclusion Effective communication is an integral part of clinical medicine. According to the modified SAIL assessment tool letters were not effectively communicating clinic details to primary care physicians posing a threat to patient care at the interface of paediatric primary and secondary care. Regular education and a pre designed layout positively impacts the quality of our written communication. Further steps need to be taken to ensure the continuity of standards in a centre where NCHDs rotate frequently.

\section{GP122 IS TREATMENT WITH ATROPINE 0.01\% EYEDROPS SAFE TO PREVENT THE PROGRESSION OF CHILDHOOD MYOPIA?}

\begin{abstract}
1,2 Jose Manuel Siurana, ${ }^{1}$ Georges Akel*, 'Lluis Cavero, ${ }^{1}$ Anna Mones, ${ }^{1}$ Ezequiel Anabalon, ${ }^{1}$ Paula Sol Ventura. ${ }^{1}$ Fundacio Hospital de Nens, Barcelona, Spain; ${ }^{2}$ Autonomous University, Barcelona, Spain
\end{abstract}

\subsection{6/archdischild-2019-epa.187}

Introduction In 2016 the effectiveness of atropine eyedrops in preventing the progression of myopia in children was confirmed. Atropine produces parasympathetic inhibition because avoids the acetylcholine fixation. Atropine's main indication is the cardiac stimulation in vagal bradycardia. Its principal cardiovascular side effects are: palpitations, tachycardia and atrial arrhythmias. A paradoxical bradycardia has been documented when atropine is administered at lower doses $(<0.1 \mathrm{mg})$. However, this concept has now been refuted. The last guidelines of the American Heart Association for Cardiopulmonary Resuscitation recommend to eliminate the minimum dose restriction of atropine during emergency intubation (class IIb). The main reluctance of parents to use atropine eyedrops in the control of myopia of their children is the fear of the cardiac effect.

Objective To evaluate the presence of cardiovascular changes after treatment with $0.01 \%$ atropine eyedrops administered to reduce the progression of myopia in children.

Material and methods Prospective observational study in 54 patients at the Nens Hospital Foundation in Barcelona (Spain) between 2016-2018. Patients received one drop of atropine $0.01 \%$ in each eye (total dose: $0.01 \mathrm{mg}$ ) with one minute of tear duct occlusion. Two analyzes were performed: before starting atropine and after 3 months of treatment. The parameters assessed were: somatometric data (weight and height), constants (heart rate and blood pressure), electrocardiographic data (P wave axis, PR segment and arrhythmias) and ultrasound data (left ventricle tele-diastolic diameter).

Results The average age was 10.2 years, with a higher percentage of women $(68.5 \%$ vs $31.5 \%)$. Only one patient presented symptoms of palpitations and there was only one case with arrhythmias. It was an 8 years old girl who presented premature atrial beats. There were no significant differences in the studied variables, with the exception of heart rate. The mean heart rate decreased from $79.4 \mathrm{bpm}$ to $75.3 \mathrm{bpm} \mathrm{(p}$ $<0.05)$.

Conclusion The use of atropine $0.01 \%$ eyedrops to prevent the progression of childhood myopia is cardiologically safe. Daily use of very low doses of atropine $(0.01 \mathrm{mg})$ over a period of 3 months results in a significant decrease in heart rate.

\section{GP123 ALTERED SYSTEMIC INFLAMMATORY RESPONSE IN PAEDIATRIC MILD TRAUMATIC BRAIN INJURY}

${ }^{1,2}$ Emer Ryan*, ${ }^{1}$ Eimear Duff, ${ }^{1}$ Dean Huggard, ${ }^{3}$ Mark Bates, ${ }^{4}$ Derek G Doherty, ${ }^{4}$ Ashanty M Melo, 1,2,5,6Eleanor Molloy. 'Discipline of Paediatircs, Department of Medicine, Trinity College, Dublin, Ireland; ${ }^{2}$ Tallaght University Hospital, Dublin, Ireland; ${ }^{3}$ Trinity Translational MedicDepartment of Surgery, Trinity Translational Medicine Institute, Trinity College, Dublin, Ireland; ${ }^{4}$ Trinity Translational Medicine Institute, Trinity College, Dublin, Ireland; ${ }^{5}$ National Childrens Reasrhc earch Centre, Dublin, Ireland; ${ }^{6}$ The Coombe Women's Hospital, Dublin, Ireland

\subsection{6/archdischild-2019-epa.188}

Aims To evaluate systemic inflammation in TBI by exploration of the inflammasome pathway, a component of the innate immune system that regulates and induces inflammation. We examine the pathway at baseline in TBI compared with healthy control children, and in vitro in response to both LPS stimulation and melatonin therapy. Melatonin has protective effects against NLRP3 inflammasome activation and has therapeutic implications.

Methods Whole blood was sampled from children with TBI $(n=10)$ within 24 hours of injury and compared to healthy age-matched controls $(n=8)$ at baseline, following stimulation with bacterial endotoxin (LPS) $(10 \mathrm{ng} / \mathrm{ml})$ and melatonin treatment $\left(10^{-3} \mathrm{M}\right)$.

Granulocytes were delineated as CD66b+ and FSC, SSC-A. Measurements of mean channel fluorescence (MCF) of CD11b and TLR4 expression on FACS Canto II were recorded and analysed with FloJo software v10. Gene Expression of NLRP3 via rtPCR was recorded in 10 patients and 10 controls at baseline and following LPS and melatonin treatment.

Results Granulocyte CD11b expression was lower in children with TBI compared to controls $(p=0.04)$ Both upregulated CD11b with LPS stimulation. Melatonin significantly decreased this LPS upregulation. There was no significant difference in baseline TLR4 expression between TBI and controls, but LPS upregulation of TLR4 was decreased by melatonin in the TBI cohort. Inflammasome was upregulated via NLRP3 expression in children with TBI compared to controls $(p=0.02)$. Melatonin significantly decreased LPS-induced upregulation of NLRP3 only in controls.

Conclusion Inflammation is altered in TBI compared to controls with altered responsiveness to melatonin treatment following LPS stimulation. The inflammasome is downregulated in children immediately following TBI. Selective inhibition of systemic inflammation targeting the inflammasome may have a future immunomodulatory role as a target in treating TBI.

\section{GP124 IL1- $\beta$ LEVELS AT PRESENTATION CORRELATE WITH SYMPTOM BURDEN AT 2 WEEKS IN PAEDIATRIC MILD TRAUMATIC BRAIN INJURY}

${ }^{1,2}$ Emer Ryan*, 'Lynne Kelly, ${ }^{1}$ Eimear Duff, ${ }^{3}$ Mark Bates, ${ }^{2}$ Turlough Bolger $1,2,4,5$ Eleanor Molloy. ${ }^{1}$ Discipline of Paediatrics, Department of Medicine, Trinity College, Dublin, Ireland; ${ }^{2}$ Tallaght University Hospital, Dublin, Ireland; ${ }^{3}$ Department of Surgery, Trinity Translational Medincine Institute, Dublin, Ireland; ${ }^{4}$ National Children's Research Centre, Dublin, Ireland; ${ }^{5}$ Coombe Women's Hospital, Dublin, Ireland

\subsection{6/archdischild-2019-epa.189}

Aims To evaluate components of the innate immune system, the inflammsome, in mild Traumatic Brain Injury (TBI) and the correlation with symptom burden 2 weeks from injury. We examine activation of the pathway at presentation, and 